\title{
Magnetisation transfer analysis and the disability resulting from multiple sclerosis
}

Over the past 20 years MRI techniques have given wonderful insights into the pathogenesis of multiple sclerosis. We have learned how lesions evolve and resolve, that measures of neuronal and axonal loss show that multiple sclerosis is not just a disease of myelin, and that over time the brain atrophies. The suspicion that the normal appearing white cell matter between the lesions of multiple sclerosis is abnormal has been confirmed. The contribution of MRI to clinical trials of novel therapeutic agents has been decisive and much more reproducible than effects on clinical measures of disability.

The Milan Neuroimaging Research Group has been remarkably productive over the past few years, studying the application of new MRI techniques to multiple sclerosis. The paper by Rovaris et al in this issue (pp 723-727) ${ }^{1}$ adds to their impressive output. Analysis of brain and cervical cord magnetisation transfer ratio (MTR) histograms in individual patients with relapsing and remitting, secondary progressive, and primary progressive multiple sclerosis has been performed in individual patients. Magnetisation transfer ratio analysis is a technique which has been developed recently. ${ }^{2}$ In a tissue the amount of magnetisation transfer depends on the capacity of macromolecular protons to exchange magnetisation with surrounding water protons and may be quantified by calculating MTR. The main influences on the value in the white matter of the brain are thought to be the myelin and water content. Loss of myelin or increased tissue water results in a reduction of MTR. A lucid explanation of the complex issues may be found in the article by van Buchem et al. ${ }^{3}$

In previous MRI studies of the cervical cord in multiple sclerosis spinal cord atrophy has been shown to correlate with physical disability to a greater extent than brain pathology. Stevenson et $a l^{4}$ demonstrated increasing atrophy over a period of follow up.

The paper by Rovaris et $a l^{1}$ indicates that both brain and spinal cord MTR measures were significantly associated with a higher probability for patients to have secondary progressive multiple sclerosis and to have difficulty in walking. Unfortunately the method does not allow an accurate estimate of the relative contributions of brain and spinal cord pathology to disability to be made. Magnetisation transfer analysis is of interest but is likely to be confined to research assessments and is unlikely to be widely applied in clinical trials. With the continuing development of MRI hardware and software, this is a rapidly evolving field. Standards for collaborative studies are under continuous appraisal and review.

S A HAWKINS

Institute of Clinical Science,

The Queen's University of Belfast,

Room 125, Mulhouse Building,

Grosvenor Road, Belfast BT12 6Bf,

Northern Ireland, UK

s.hawkins@qub.ac.uk

1 Rovaris M, Bozzali M, Santuccio G, et al. Relative contributions of brain and cervical cord pathology to multiple sclerosis disability: a study with magnetisation transfer ratio histogram analysis. I Neurol Neurosurg Psychiatry 2000;69:723-7.

2 Grossman RI. Application of magnetization transfer imaging to multiple sclerosis. Neurology 1999;(suppl 3):S8-11.

3 Van Buchem MA, McGowan JC, Grossman RI. Magnetization transfer histogram methodology: its clinical and neuropsyclological correlates. Neurology 1999;53 (suppl 3):S23-8.

4 Stevenson VL, Leary SM, Losseff NA, et al. Spinal cord atrophy and disability in MS. A longitudinal study. Neurology 1998;51:234-6.

\section{Unmet needs in multiple sclerosis: the role of community services}

Despite significant diagnostic and therapeutic advances in the past decades, multiple sclerosis still results, for most of the patients, in different degrees of disabilities that tend to progress over time, requiring various medical and nonmedical interventions. Due to the variability in disease evolution, frequent assessments of patients' health status are necessary to provide the best management of their needs.

High quality medical care, which usually requires expensive diagnostic and therapeutic procedures and the patient's admissison to hospital, should be supplemented by reabilitation programmes and social interventions aimed at preventing disabilities and promoting the patient's independence in his social environment.

This global approach is mandatory to provide patients with the best care and it may result, at the same time, in an optimal use of resources, thus contributing to cost containment.

Valuable indicators, which health care planning should be based on, can be drawn from surveys on utilisation of medical and social services.

This issue is considered by Freeman and Thompson ${ }^{1}$ in this issue ( $p$ p 728-732) who investigate the current use of services and home modifications in a cohort of 150 patients with multiple sclerosis with a broad range of disease severity.

The study shows that a large proportion of patients with multiple sclerosis, even those severely disabled, fail to receive any community service, thus suggesting that many of the patients' needs are unmet by the current clinical practice. 
Althought derived from a specific setting, the authors' conclusions fit with others' clinical experience and are likely to be true also in different sociocultural contexts.

It may be asked whether physicians are really aware of the actual needs of their patients, as it has been shown that patients' and doctors' perceptions of multiple sclerosis disability diverge considerably. ${ }^{2}$

More likely, in my opinion, the poor coordination between doctors and other health and social care professionals is responsible for the inadequate management of patients with multiple sclerosis. Interestingly a previous study found that, in severely disabled patients, unmet needs were consistently higher in those whose sole contact was with health professionals compared with those reassessed on a multidisciplinary basis by health and social services operators. ${ }^{3}$ It is expected that the improvement in utilisation of community services will result in cost containment by limiting the need of hospital admittance.

This is particulary important for severely disabled patients with multiple sclerosis who primarily account for the highest charges to the healthcare system. ${ }^{4}$
Most importantly healthcare givers and authorities should bear in mind that an effective coordination between professional operators and available services may contribute to improve the quality of life of patients with multiple sclerosis no less than the use of new expensive therapeutic agents does.

Careful cost effectiveness studies of alternative forms of treatment should be encouraged to provide clinical guidelines to help clinicians in their everyday practice.

C MILANESE

Instituto Neurologico Carlo Besta, Via Celoria 11, 20133 Milano, Italy

msgroup@istituto-besta.it

1 Freeman JA, Thompson AJ. Community services in multiple sclerosis: still a matter of chance. $\mathcal{F}$ Neurol Neurosurg Psychiatry 2000;69:728-32.

2 Rothwell PM, McDowell C, Wong CK, et al. Doctors and patients don't agree: cross reactional study of patients and doctors perceptions and assessement of disability in Multiple Sclerosis. BMF 1997;314:1580-3.

3 Williams $M H$, Bowie C. Evidence of unmet need in the care of severely physycally disabled adults. BMF 1993;306:95-8.

4 Stolp-Smith KA, Atkinson EJ, Campion ME, et al. Health care utilization in multiple sclerosis. A population based study in Olmsted County, NM. Neurology 1998;50:1594-600.

\section{Hippocampal sclerosis: cause or consequence of febrile seizures}

Retrospective studies of patients who underwent surgery for refractory temporal lobe epilepsy found a relation between history of early febrile seizures, hippocampal sclerosis, and the development of temporal lobe epilepsy. ${ }^{1}$ Previous MRI studies have reported smaller volumes of the amygdala and the hippocampus in patients with prolonged febrile seizures in childhood than in those without such a history ${ }^{2}$ suggesting that early febrile seizures are a precipitating event that leads to hippocampal sclerosis. In prospective studies, early febrile seizures or other brain injuries rarely lead to hippocampal sclerosis and temporal lobe epilepsy. ${ }^{3}$ Although epidemiological studies have not yet corroborated the "surgical" correlations, there is convincing evidence that children with complicated febrile seizures have a higher risk for development of temporal lobe epilepsy. The precise pathophysiological relation, however, between febrile seizures, hippocampal sclerosis, and temporal lobe epilepsy remains controversial. Controversy exists as to whether febrile convulsions cause hippocampal sclerosis, and that this in turn is responsible for temporal lobe epilepsy, or hippocampal sclerosis develops independently, or whether there is a pre-existing pathology which predisposes children to have febrile seizures and, later on hippocampal sclerosis. Bower et $a l^{4}$ in this issue (pp 733744) report their quantitative MRI findings in 77 patients with histologically proved hippocampal sclerosis. By contrast with previous reports, they found that hippocampal atrophy determined by MRI is no more severe in patients with temporal lobe epilepsy with a history of febrile seizures compared with those without such a history. The high proportion $(40 \%)$ of patients with febrile seizures in this study suggests an association between febrile seizures and hippocampal sclerosis, but the severity of hippocampal atrophy determined by MRI is independent of a history of febrile seizures. This is to be expected if early febrile seizures are a marker of an underlying cerebral injury that is associated with hippocampal sclerosis and not the precipitating injury itself. Their conclusion, however, that hippocampal sclerosis is a pre-existing abnor- mality providing a substrate for febrile seizures, is not supported by their data or cited publications.

Retrospective surgical series have various limitations: the selection bias in surgical series could obscure a correlation between MRI based hippocampal volume measurements and history of febrile seizures and other clinical variables; although MRI volume measurements are related to the severity of hippocampal sclerosis, these MRI parameters are not entirely explained by variations in neuronal and glial densities in the hippocampal subregions ${ }^{5}$; correlation analyses do not provide direct evidence for the existence or non-existence of a causal relation between febrile seizures and severity of hippocampal atrophy; the difficulty in recording a history of febrile seizures without a maternal history and in distinguishing complex from simple febrile seizures is well recognised.

The number of complex febrile seizures in this and previous studies ${ }^{2}$ is too small to draw meaningful conclusions. Based on the predominance of simple febrile seizures in their study and recent MRI findings in a study of familial febrile seizures, ${ }^{6}$ the authors question the assumption that brains of infants with simple febrile seizures are normal. These findings suggest that some pre-existing brain injury predisposes patients to develop febrile seizures and contribute to the development of subsequent hippocampal sclerosis. Hippocampal sclerosis is not selective to patients with a history of early febrile seizures and multiple injuries may in fact be necessary for the development of hippocampal sclerosis, such as the occurrence of febrile seizures, head trauma, meningits, or encephalitis in the presence of some pre-existing, possibly developmental abnormality. Volumetric MRI may miss those subtle developmental abnormalities.

Bower $e t a l^{4}$ and others ${ }^{2}$ did not find a relation between hippocampal atrophy and duration of temporal lobe epilepsy, seizure frequency, and other clinical variables. These findings did not suggest continuing hippocampal or amygdala atrophy during chronic temporal lobe epilepsy that could be detected by volumetric MRI, but did not 